\title{
Reformation und Bauernkrieg
}

\section{Sabrina Pilsinger}

Kerngebiet: Österreichische Geschichte

eingereicht bei: Priv.-Doz. Dr. Robert Rebitsch und

Univ.-Prof. Mag. phil. Dr. rer. nat. Kurt Scharr

eingereicht im: SoSe 2019

Rubrik: Seminar-Arbeit

\begin{abstract}
Reformation and Peasants' War

This paper examines the connections and interactions between the Reformation and the German Peasants'War. It focusses on the central actors of the two processes and shows the personal connections between the leaders of the different groups of reformation, i.e. Martin Luther and Ulrich Zwingli, and as well as the process of the uprising and the demands of the peasants. In addition, the aspect of space and the variety of demands will be addressed.
\end{abstract}

\section{Einleitung}

„Widerstand lert uns erst recht erkennen, was glouben ist, was recht gebättet ist."1

Die Bauernkriege und die Reformation sind zwei historische Elemente, die gern, trotz ihres längerfristigen Charakters, als Großereignisse des frühneuzeitlichen Europas gesehen werden - als Zeitenwenden. Das Jubiläum der Reformation, welches 2017 den 500. Jahrestag des Thesenanschlags Martin Luthers feierte, wurde bereits ausgiebig zelebriert. Das Jahr, in dem das Gedenken auf den sogenannten deutschen bzw. auf den

Ulrich Zwingli, Wer Ursache gebe zu Aufruhr usw. (7. Dezember resp. 28. Dezember 1524), in: Emil Egli (Hrsg.), Huldreich Zwinglis sämtliche Werke, Bd. 3, Leipzig 1914, [http://www.irg.uzh.ch/static/zwingli-werke/index. php?n=Werk.42], eingesehen 25.6.2019. In der Arbeit wird die Schreibweise Ulrich Zwingli verwendet. Die Autorin ist sich aber bewusst, dass es weitere Varianten gibt. 
oberösterreichischen Bauernkrieg gelenkt wird, steht 2025 bevor. Diese massenmedial gesteuerte Form der Erinnerung lässt uns häufig vergessen, dass die Reformation und die bäuerlichen Aufstände eine Vor- und Nachlaufzeit hatten. Es waren einschneidende Entwicklungen, aber keine punktuellen Begebenheiten und schon gar keine plötzlichen Ereignisse, die Naturgewalten ähnelten. ${ }^{2}$ Beide sind zwar als eigenständige Prozesse zu betrachten, aber dennoch sind die Bauernkriege und die Reformation in ihrer Basis miteinander verquickt. Kein Wunder, denn die Reformation gab mehr oder weniger die Leitfragen für die nächsten Jahrhunderte vor.

Die Erforschung des Bauernkrieges erfordert eine Auseinandersetzung mit der Reformation. Behandelte Themen sind die direkten Äußerungen Martin Luthers zum Thema, die Rolle von reformatorischen Predigern oder die intensiv religiös motivierten Persönlichkeiten im Bauernkrieg. Letztere hatten ebenso starke soziale wie wirtschaftliche Motive, sich zu erheben. Dennoch waren es religiöse Gründe, die in ihren Forderungen höchste Priorität hatten. ${ }^{3}$ Trotzdem gab es für jene Vorwürfe, die angeführten Gründe wären nur schönes Beiwerk, um die eigentlich wirtschaftlich motivierten Absichten voranzubringen, Nährboden.

Schon lange beschäftigen sich Historiker*innen mit den Verbindungen zwischen Reformation und Bauernkrieg. Je nachdem, aus welcher historischen Disziplin sie kommen, wurde bzw. dem Bauernkrieg einmal mehr, einmal weniger religiöser Gehalt zugesprochen. Für Wilhelm Zimmermann ${ }^{4}$ etwa waren zwar beide Ereignisse verknüpft, die Revolution stand jedoch im Vordergrund. Er sah die Forderungen mehr in ein „religiöses Gewand" gewickelt. Spätere Historiker*innen sahen einen eindeutigeren religiösen Zusammenhang. Dietrich Lösche sieht zum Beispiel die Verbindung darin, dass beide Bewegungen theologische Kritik an den geistlichen Fürstentümern in Deutschland übten. ${ }^{5}$ Bei Heiko Obermann wird die Verknüpfung noch deutlicher:

„Das neue reformatorische Ferment bewirkte nun eine politische Radikalisierung im Sinne eines biblisch-theologisch begründeten geistigen Widerstands gegen die diesseitige Machtfülle der Kirche, ihre Steuerprivilegien in Stadt und Land und ihre Rom-orientierte Wirtschaftspolitik."

Es ergeben sich die allgemeinen Fragen: Wie sind die Forderungen der Bauern und Bäuerinnen zu sehen: wirtschaftspolitisch oder theologisch-religiös? Oder ist die Kombination aus beiden möglich? Folgende Fragen für die Arbeit ergeben sich daraus: (1) Inwieweit ist der Vorwurf gerechtfertigt, die reformatorischen Ideen seien nur ein Vehikel gewesen, um wirtschaftliche Interessen der Bauern und Bäuerinnen durchzuset-

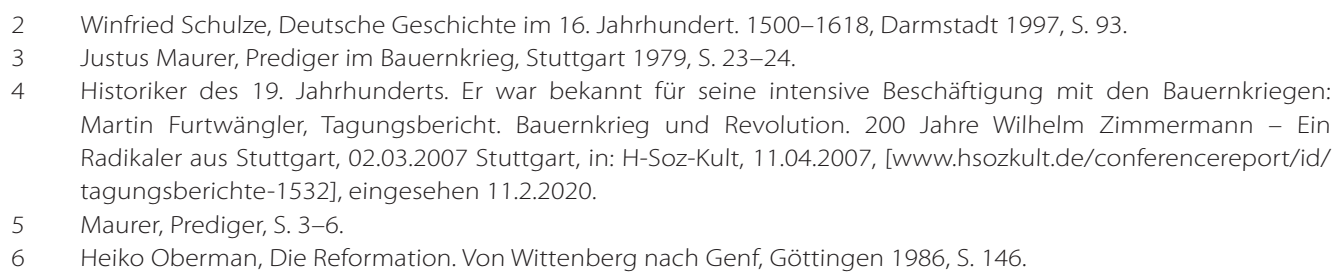


zen? (2) Welche Gemeinsamkeiten und Wechselwirkungen gab es zwischen Reformation und Bauernkrieg?

Hierauf bauen die Thesen auf: (1) Obwohl der Vorwurf, nur eine wirtschaftliche Strategie zu verfolgen, eine beliebte Argumentationslinie ist, kann die Wichtigkeit der reformatorischen Ideen nicht von der Hand gewiesen werden. Sie können nicht nur zweitrangig neben wirtschaftlichen Interessen genannt werden. (2) Reformation und Bauernkrieg fungierten füreinander als Motoren für die Generierung von Ideen und für deren praktische Umsetzung. Es ist die Absicht dieser Arbeit, dies anhand der Akteursebene deutlich zu machen.

Aufbauend auf diese Fragestellungen wird die Seminararbeit in drei Großkapitel unterteilt. Im ersten wird ein historischer Überblick über Reformation und Bauernkriege gegeben. Hier sollen vor allem die Vorgeschichten und erste Querverbindungen herausgearbeitet werden. Diese sind ebenso auf der personellen Ebene zu finden, weshalb zweitens auf Prediger und ihre Wirkung eingegangen wird - wobei Martin Luther, mit seinen Schriften, sowie Ulrich Zwingli, mit seinem Einfluss auf den süddeutschen Raum, besondere Aufmerksamkeit zukommt. Auch Thomas Müntzer wird gesondert behandelt. Dies wird damit begründet, dass er als religiöser Gegenspieler zu Luther fungierte. Hierbei wird der deutlich vernetzte Charakter der Konfliktebenen dargestellt. Drittens wird das Element des Raumes näher behandelt. Durch die Lektüre der neuesten Literatur und vor allem durch die angeregten Diskussionen des Seminars wurde deutlich, wie individuell die Gebiete in ihren sozialen, wirtschaftlichen und vor allem religiösen Strukturen waren. Schlussendlich folgt das Fazit.

Die Literatur zum Thema Reformation bzw. Bauernkriege ist nicht leicht zu überblicken, da die Thematiken doch recht verwoben sind. Dennoch kann die Forschungsliteratur grob in vier Blöcke eingeteilt werden: (1) Literatur, die sich primär mit den bäuerlichen Aufständen während des 16. Jahrhunderts beschäftigt, ${ }^{7}$ (2) Literatur, die sich vor allem mit den bäuerlichen Aufständen während des 17. Jahrhunderts beschäftigt, ${ }^{8}$ (3) Literatur, die sich mit der Reformation beschäftigt, in der die Bauerkriege aber ein Randthema bleiben, sowie (4) Literatur, die die Reformation in direkten Kontext mit den Bauerkriegen setzt. ${ }^{9}$ Die Blöcke eins und zwei reißen die Thematik Bauernkrieg und Reformation sehr wohl an, jedoch bleibt die Neuorientierung des Glaubens meist nur ein Aspekt

$7 \quad$ Hans-Ulrich Wehler (Hrsg.), Der Deutsche Bauernkrieg 1524-1526 (Geschichte und Gesellschaft Sonderheft 1), Göttingen 1975; Adolf Laube/Hans Werner Seiffert (Hrsg.), Flugschriften der Bauernkriegszeit, Wien 1978; Peter Blickle, Der Bauernkrieg, München $2011^{4}$.

8 Amt der oberösterreichischen Landesregierung, Abteilung Kultur (Hrsg.), Der oberösterreichische Bauernkrieg 1626, Katalog zur Ausstellung im Linzer Schloss und im Schloss zu Scharnstein im Almtal von 14.05.197631.10.1976, Linz 1976; Georg Heiligsetzer, Der oberösterreichische Bauernkrieg 1626 (Militärhistorische Schriftenreihe Heft 32), Wien 1976; Volker Lutz, Der Aufstand von 1596 und der Bauernkrieg von 1626 in und um Steyr (Veröffentlichung des Kulturamtes der Stadt Steyr Folge 33), Steyr 1976; Willi Flicker, Der oberösterreichische Bauernkrieg von 1626 in seinem politischen, konfessionellen, sozialen und kulturellen Umfeld, Dipl. Innsbruck 1991.

9 Peter Barton (Hrsg.), Sozialrevolution und Reformation. Aufsätze zur Vorreformation, Reformation und zu den Bauerkriegen in Südmitteleuropa, Wien-Köln-Graz 1975; Reiner Wohlfeil (Hrsg.), Bauernkrieg 1524-25. Bauernkrieg und Reformation (Nymphenburger Texte zur Wissenschaft 21), München 1975; Justus Maurer, Prediger im Bauernkrieg, Stuttgart 1979 
unter vielen. ${ }^{10}$ In Block drei werden häufig die Aussagen Luthers zu den Bewegungen untersucht. ${ }^{11}$ Andere, für die bäuerlichen Bewegungen relevante Reformatoren, werden ebenfalls behandelt. ${ }^{12}$ Block vier ist der wichtigste für diesen Beitrag.

\section{Reformation und Bauernkrieg im historischen Überblick}

Dass die Bauernkriege sowie die Reformation eigenständige Entwicklungen waren, jedoch eine nicht zu leugnende Verbindung besaßen, wurde schon in der Einleitung betont. Doch wo liegen die Gemeinsamkeiten, wo die Wechselwirkungen? Zunächst muss die Vorgeschichte der beiden Ereignisse beleuchtet werden. Sowohl den bäuerlichen Aufständen 1525 als auch der Reformation gingen soziale Unruhen und das Aufflackern von kirchlichem Ungehorsam voran. Im 15. Jahrhundert waren dies für Bauern und Bäuerinnen der „arme Konrad” oder die Bundschuh-Bewegungen, für die Reformation die vielen Frömmigkeitsbewegungen. Schon die Bundschuh-Bewegungen in Südwestdeutschland beinhalteten Forderungen, die im sogenannten „großen deutschen Bauernkrieg" von 1525 erneut Wichtigkeit erlangten. Dazu gehörten zum Beispiel die Abschaffung von Abgaben und die Freigabe von Jagdrechten. Ein ebenso großer Punkt war jedoch die Ablehnung der sogenannten „Pfaffen- und Klösterherrschaft“13. Damit verfügten nicht nur die weltlichen Herrscher*innen über Menschen und deren Arbeitskraft.

Das 15. Jahrhundert, also die Vorläuferzeit beider Ereignisse, ist von Gegenläufigkeit, von Differenzierung und Zentrierung geprägt. Individualität und Subjektivität wurden gesteigert. Es entstand eine Pluralität von auseinanderdriftenden Gruppierungen, verbunden mit starkem Autoritätsverfall kirchlicher Machtträger. ${ }^{14}$ Die Zeit war auch geprägt von technologischen Revolutionen wie der Verbreitung und Etablierung des Buchdruckes. Dadurch kam es zu einer verstärkten Vernetzung der Gesellschaft, Mobilität und der Austausch von Informationen wurden verbessert und gefördert. Die Innovation der Bauernbewegungen um 1524/25 waren nicht unbedingt ihre Forderungen, diese befanden sich schon länger im Umlauf, sondern deren Begründung. Sie griffen das biblische Recht des Evangeliums auf. Damit nahmen die Bäuerinnen und Bauern Anstöße aus der Reformation an und setzten sie selbstständig um. ${ }^{15}$ Dies führte zur Formulierung der Zwölf Artikel, des Grundprogramms der aufständischen Bäuerinnen und Bauern, die sich wie ein roter Faden durch den deutschsprachigen Raum zogen. Sie enthielten reformatorisches Gedankengut und legitimierten sich unter Berufung auf das Evangelium. ${ }^{16}$

10 Etwa: Flicker, Der oberösterreichische Bauernkrieg.

11 Paul Althaus, Luthers Haltung im Bauernkrieg, Darmstadt 1971

12 Peter Blickle (Hrsg.), Zwingli und Europa. Referate und Protokoll des internationalen Kongresses aus Anlass des 500. Geburtstages von Huldrych Zwingli vom 26.-30. März 1984, Göttingen 1985.

13 Karl Heinz zur Mühlen, Reformation und Gegenreformation, Göttingen 1999, S. 115-116.

14 Berndt Hamm, Bürgertum und Glaube. Konturen der städtischen Reformation, Göttingen 1996, S. 73-76.

15 Zur Mühlen, Reformation und Gegenreformation, S.116.

16 Ebd., S. 117 
Am Anfang des Jahres 1525 konnten die Bauernbewegungen und -aufstände sogar militärische und politische Erfolge erzielen. Teilweise wurden Vereinbarungen und Zugeständnisse gemacht. Dennoch kam es anderenorts zu blutigen Gemetzeln, Übergriffen und sogar Plünderungen. Die anfänglichen Vermittlungsversuche auf Seiten der Herrscher*innen wichen immer mehr einer Vernichtungsstrategie. Allmählich gingen die Aufstände zu Ende: Im Reichsgebiet im Sommer 1525, im Alpenraum hielten die Bewegungen bis 1526 durch. ${ }^{17}$ Während sich die von der marxistischen Geschichtsschreibung sogenannte „frühbürgerliche Revolution“ nicht durchsetzen konnte, ging die Reformation weiter. Trotz Differenzen in verschiedenen Lagern, etwa unterschiedlichen Lehrmeinungen von Zwingli und Luther, konnten sich die neuen religiösen Lehren im Laufe des 16. Jahrhunderts etablieren und schlussendlich in bestimmten Gebieten durchsetzen. Dies geschah wahrscheinlich auch durch die Unterstützung des Adels. ${ }^{18}$

Die wohl wichtigste Quelle, neben den Schriften der Reformatoren, sind die Zwölf Artikel. Sie bildeten das Grundprogramm der Aufständischen. Der erste Artikel und damit wohl die zentralste Forderung war die nach der Wahl des eigenen Pfarrers. Die anderen Artikel, die zwar weniger religiös waren und mehr wirtschaftliche Forderungen beinhalten, bezogen sich immer noch auf das göttliche Recht aus der Bibel. ${ }^{19}$

Bauernkrieg und Reformation gehören zu jenen Ereignissen der deutschen Geschichte, in denen traditionelle politische Verhaltensmuster in Frage gestellt wurden und Bevölkerungsschichten selbst nach Lösungsmöglichkeiten für ihre Probleme suchten. ${ }^{20}$ Es ist anzunehmen, dass Reformation und Bürgerkrieg deshalb auch beinahe zeitgleich entstehen konnten, da wirtschaftliche, politische, staatliche und religiöse Veränderungen sich zu Krisen zugespitzt hatten. ${ }^{21}$ Beides sind Phänomene, die Massen in Bewegung versetzten und hochemotional aufgeladen waren. Die Geschichtswissenschaft weist auf die Verbindung der beiden bzw. auf die Bedeutung des jeweils anderen hin. Dennoch wirkt es gerade in der marxistischen Geschichtsschreibung so, als versuche sie eine Entkopplung von Religion und Bauernkrieg zu erreichen. Zwar ist es durchaus möglich, beide Phänomene getrennt, unter verschiedenen Teilaspekten, zu betrachten, dennoch sollte betont werden, wie sehr sie sich gegenseitig beeinflussten. Wichtig ist es festzustellen, dass es nicht die eine Reformation gab, sondern viele Teilperspektiven, die auf Erneuerung aus waren. Ähnlich verhält es sich mit den Bauernkriegen: Es gab eine große Bandbreite an Argumentationen und Beweggründen.

17 Heinrich Lutz, Reformation und Gegenreformation (Oldenbourg Grundriss der Geschichte 10), München 1974, S. 35-36.

18 Ebd., S. 37

19 Olaf Mörke, Die Reformation. Voraussetzungen und Durchsetzung (Enzyklopädie deutscher Geschichte 74), München 2005, S. 36.

20 Heinrich Sperling, Möglichkeiten zu sozialem und politischem Lernen in der deutschen Geschichte. Eine Untersuchung des Bauernkrieges, in: Rainer Wohlfeil (Hrsg.), Der Bauernkrieg 1524-26. Bauernkrieg und Reformation, München 1975, S. 246-279, hier S. 248.

21 Laube/Seiffert (Hrsg.), Flugschriften, hier S. 11 


\section{Prediger und ihre Wirkung}

Um das Argument der vielseitigen Beweggründe aus dem letzten Kapitel zu vertiefen, wird ein Blick auf die religiösen Köpfe der Reformation geworfen. Prediger waren eine der einflussreichsten Gruppen in der Zeit des 16. Jahrhunderts. Sie waren mobil, waren des Lesens kundig, kannten sich mit religiösen Fragen aus und wurden meist geachtet. In einer Gesellschaft, in der das Wort Gottes und die Welt des Sakralen einen höheren Stellenwert als heute einnahmen, waren sie es, die Informationen weitergaben, die öffentliche Stimmung beeinflussten und diese bis zu einem gewissen Grad lenken konnten. ${ }^{22}$ In der konfliktreichen Zeit der Bauernkriege um 1525 waren ihre Haltungen gegenüber den Aufständen durchaus ambivalent. Reformatorische Prediger waren sowohl Förderer als auch Gegner des zivilen Aufstandes. Die Nähe zur Reformation, zum Beispiel in den Zwölf Artikeln, war paradoxerweise Nährboden für Kritik an der Ehrlichkeit der Bewegungen. Waren die reformatorischen Ideen nur Mittel, um wirtschaftliche Forderungen durchzusetzen, oder tatsächlich religiös wichtig? Es handelte sich um ein widersprüchliches Beziehungsgeflecht.

Es wird deshalb exemplarisch auf Personen eingegangen, welche während dieser Zeit große Wirkungsgebiete hatten, welche die Reformation vertraten und direkten Einfluss auf Obrigkeit und Bauernschaft ausübten. Faszinierenderweise sahen schon diese Zeitgenoss*innen die Zusammenhänge der Bewegung in einer Weise, wie es auch die frühe Geschichtswissenschaft bereits tat. Dies lässt den Schluss zu, dass sich diese Personen über ihren Einfluss im Klaren waren und diesen in ihrem Interesse zu steuern wussten. Im Zuge der vorliegenden Arbeit werden drei dieser einflussreichen Persönlichkeiten herangezogen: Martin Luther, der zwar ein theologisches Konzept stellte, aber die Umsetzung der Bäuerinnen und Bauern ideologisch kritisierte, weiters Ulrich Zwingli, der eine Art Mediatorenrolle innehatte, sowie drittens Thomas Müntzer, welcher als Agitator der Aufstände fungierte.

\subsection{Martin Luther}

Auch wenn andere reformatorische Prediger die Bauernaufstände mehr gefördert, unterstützt und religiös untermauert hatten, ist es Martin Luther, der wohl am intensivsten mit dem Thema verbunden wird. Zu sehr ist seine Lehre vom "reinen Wort Gottes"23 damit assoziiert. Selbst bei einer oberflächigen Recherche zum Thema Bauernkrieg und Reformation finden sich unweigerlich die Schriften Luthers, der als Zeitgenosse über die Ereignisse schrieb. Zahllose Publikationen befassen sich mit Veröffentlichung, Aufbau und Auslegung seiner Standpunkte.24 Auch muss ausdrücklich erwähnt wer-

22 Zur Wichtigkeit der Prediger: Maurer, Prediger.

23 Hier bezogen auf ein Alleinstellungsmerkmal der protestantischen Theologie. Die Bibel ist der Kern und die Quelle des Glaubens. Die Autorität anderer Dogmen wird nicht anerkannt: Luther 2017, 0. D., [https://www.luther2017. de/de/reformation/und-kultur/bild-bibel/sola-scriptura/index.html], eingesehen 12.2.2020.

24 Hierzu, im Zusammenhang der Bauernkriege, etwa: Althaus, Luthers Haltung; Michael Beyer, Die drei Bauernkriegsschriften Martin Luthers von 1525, in: Werner Greiling/Thomas Müller/Uwe Schirmer (Hrsg.), Reformation und Bauernkrieg (Quellen und Forschungen zu Thüringen im Zeitalter der Reformation 12), Köln 2019, S. 241-258. 
den, dass Luthers Meinung zum Thema eine längerfristige Entwicklung durchmachte. Ab Mitte des Jahres 1525 dürfte er die Zwölf Artikel gekannt haben, denn er reagierte mit der Schrift „Ermahnung zum Frieden auf die Zwölf Artikel der Bauernschaft in Schwaben" auf die Forderungen. Darin erkannte er die wirtschaftliche sowie rechtliche Not der bäuerlichen Bevölkerung an und ersuchte die Fürsten, einen Kompromiss zu suchen. Dennoch sprach er den Bäuerinnen und Bauern ab, sich auf göttliches Recht berufen zu können. Ihre Forderungen seien weltlicher Natur, also seien sie in der weltlichen Rechtsgemeinschaft zu verhandeln. Eine freie Wahl der Pfarrer gestand er einer Gemeinde nur zu, wenn die nächsthöhere Instanz ihr keinen zur Verfügung stellte. Bis zu diesem Zeitpunkt war die Haltung Luthers zwar ablehnend gegenüber der Einstellung, die Konflikte auf Basis von Theologie zu führen, doch sprach er der bäuerlichen Bevölkerung nicht ihre Not ab. Er erkannte an, dass es offensichtlich Probleme gab, diese aber nicht in der Arena des Glaubens auszutragen wären. Außerdem riet er den Herrscher*innen, sich mit den Bäuerinnen und Bauern zu einigen. ${ }^{25}$

Die Einstellung Luthers radikalisierte sich aber noch im selben Jahr dramatisch. Ausgelöst wurde dies durch seine Reise nach Eisleben. Diese erweiterte er in den Thüringer Raum, wo er den tobenden Bauernaufstand am eigenen Leib erfuhr. Verstört durch die Brutalität und heftige Realität des Konflikts wurde Luther zu seiner wohl berühmtesten Bauernschrift veranlasst: „Wider die räuberischen und mörderischen Rotten der Bauern“. Doch auch hier zeigt sich, dass Luthers literarischer Ausbruch zunächst mit einer Ermahnung zum Frieden gedruckt wurde. Dies lässt die Argumentation zu, dass er keineswegs einseitig gegen die Bauerschaft schrieb. Dass er etwa den Vertrag zwischen dem Schwäbischen Bund und den oberschwäbischen Bäuerinnen und Bauern mit Vor- und Nachwort versah und dafür plädierte, diese Lösung als Vorbild zu nehmen, spricht ebenfalls dafür, dass er eine friedliche Beendigung der Konflikte anstrebte. ${ }^{26}$ Dennoch bleiben seine Worte rigoros und extrem. Stellenweise findet sich der Aufruf nach der Niederschlagung des Bauernaufstandes. Diese Brutalität kann auch als Reaktion auf die sogenannte „Weinsberger Bluttat" gewertet werden. Diese Bluttat geschah am Ostersonntag 1525: Es wurden mehrere adlige Personen in der Stadt Weinsberg massakriert. ${ }^{27}$ Im Juli 1525 kommentierte Luther seine harsche Reaktion in der Schrift „Sendbrief vom harten Büchlein wider die Bauern" und verteidigte seinen Standpunkt. Die Bäuerinnen und Bauern hätten unrechtmäßig gehandelt, aber die Obrigkeit müsse nun gnädig mit ihnen umgehen. Ein besonderes Feindbild für Luther stellte Thomas Müntzer dar, den er in diesen Fragen als seinen Gegenspieler ansah. Er bezeichnete ihn als „mördischen und blut gyrigen propheten" und vermutete, dass der Teufel höchstpersönlich durch ihn spreche. ${ }^{28}$

25 Zur Mühlen, Reformation und Gegenreformation, S. 120-121.

26 Ebd. S. 121.

27 Thomas Müller, Bauernkrieg in Thüringen. Eine kurze rezeptionsgeschichtliche Einführung, in: Werner Greiling/ Thomas Müller/Uwe Schirmer (Hrsg.), Reformation und Bauernkrieg (Quellen und Forschungen zu Thüringen im Zeitalter der Reformation 12), Köln 2019, S. 9-20, hier S. 9.

28 Martin Luther, Eine schreckliche Geschichte und ein Gericht über Thomas Müntzer, in: Adolf Laube/Hans Werner Seiffert (Hrsg.), Flugschriften der Bauernkriegszeit, Wien 1978 S. 499-505, hier S. 499. 


\subsection{Ulrich Zwingli}

Trotz seines bedeutenden Einflusses auf die Ideen des Bauernkrieges spielt Ulrich Zwingli in der Literatur meist eine untergeordnete Rolle. Zwar nahm er eine zentrale meinungspolitische Funktion ein - vor allem die radikalen sozial-wirtschaftlichen Ideen wurden durch die bäuerliche Bevölkerung rezipiert - doch gibt es ein großes Ungleichgewicht in der Forschung und dem populären Wissen zugunsten Martin Luthers. ${ }^{29}$ Dennoch war es Ulrich Zwingli, der sich im theologischen Austausch auf die Seite der Aufständischen stellte. Auch wenn er deren gewalttätiges Vorgehen nicht guthieß, war er nicht einverstanden mit den anderen Reformatoren, die das Aufbegehren als einen Akt gegen die religiöse Ordnung des Evangeliums ansahen. ${ }^{30} \mathrm{Zu}$ bedenken ist dabei, dass die bäuerlichen Aufstände im Züricher Raum anders verliefen als etwa in Thüringen. Auch wenn es Stimmen gibt, die zu Recht darauf hinweisen, dass sich die Sphären der Einflüsse zwischen verschiedenen theologischen Ausrichtungen nicht immer konkret trennen lassen, ${ }^{31}$ war es Zwingli, der eine direkte politische Einflussnahme ausübte. Im Züricher Raum kamen schon 1523 Forderungen auf, welche denen des deutschen Bauernkrieges ähnelten, z. B. die Beschwerde über Zehentabgaben, die für zu groß gehalten wurden oder nicht im Interesse der Bevölkerung eingesetzt würden. Zwingli verfasste in dieser Zeit mehrere Schriften und fungierte als Mediator zwischen der Obrigkeit und der Bevölkerung, etwa mit den 1523 erschienenen Schriften „Von Göttlicher und menschlicher Gerechtigkeit“ und „Wer Ursache zum Aufruhr gibt“32. Dem Volk wurde zugestanden, dass es das Wort Gottes vernehmen dürfe und in weiterer Folge nicht die Obrigkeit angreifen werde. Dank dieser Zugeständnisse blieb die Lage ruhig. ${ }^{33}$ Als sich die Situation 1525 durch die deutschen Bauernunruhen zuspitzte, lag es wieder an Zwingli, moderierend einzugreifen. Die Züricher Bäuerinnen und Bauern nahmen die Forderungen der Zwölf Artikel teilweise auf. Wieder war der Zehent Diskussionspunkt, aber auch die Pfarrerwahl und die Leibeigenschaft kamen als Themen auf. Vielleicht wurde gerade durch diese Vermittlerposition der Konflikt klein gehalten, und obwohl nur ein kleiner Teil der Forderungen umgesetzt wurde, schien dies der Bevölkerung zu genügen. Der Züricher Rat ließ sich dazu bewegen, die Leibeigenschaft abzuschaffen. Offenbar linderte dies die Not der bäuerlichen Bevölkerung genug, um die Proteste

29 Dadurch, dass es zu keinem größeren Aufstand im Züricher Raum kam, sondern dieser größtenteils durch Mediation von Zwingli verhindert werden konnten, gibt es eigentlich keine weitreichende Literatur zu diesem Thema. Zwar wird manchmal erwähnt, wie wichtig seine Argumentationen für die Bauernbewegung waren, aber er bleibt eine Randfigur: Oberman, Die Reformation, S. 146-153. In Bezug auf Balthasar Hubmaier: Wilhelm Mau, Balthasar Hubmaier, Berlin-Leipzig 1912, S. 45-47. Luther hingegen wird in fast jedem Standardwerk zum Thema herangezogen: Franz Brendle, Das konfessionelle Zeitalter, Berlin 2010, S. 111-112.

30 Peter Blickle, Die Reformation im Reich, Stuttgart 2000³, S. 142-143.

31 Heinz Schilling, Die deutsche Gemeindereformation. Ein oberdeutsch-zwinglianisches Ereignis vor der „reformatorischen Wende" des Jahres 1525?, in: Zeitschrift für Historische Forschung 14 (1987), Heft 3, S. 325-332, hier S. 330-331.

32 Ch. Scheidegger, Bauern \& Bauernkrieg, 2002, [https://www.zhref.ch/themen/reformationsjubilaeum/allgemeineinformationen/huldrych-zwingli/zwingli-lexikon-von-a-bis-z-1/lexikon-b/bauern-bauernkrieg], eingesehen 17.6.2019.

33 Ulrich Zwingli, Von göttlicher und menschlicher Gerechtigkeit (30. Juli 1523), in: Emil Egli (Hrsg.), Huldreich Zwinglis sämtlicheWerke, Bd. 2, Leipzig 1908, [http://www.irg.uzh.ch/static/zwingli-werke/index.php?n=Werk.21], eingesehen 17.6.2019. 
nicht weiter fortzusetzen. ${ }^{34}$ Ähnlich wie Luther bezog sich Zwingli auf die göttliche Ordnung, die rechtens sei und nicht von Menschen bezweifelt werden solle. Direkte Äußerungen Zwinglis zu Luthers Aussagen oder Müntzer und die Situation in Mühlhausen sind bei der vorliegenden Recherche nicht aufgetaucht.

\subsection{Thomas Müntzer}

Als letzter Prediger wird Thomas Müntzer behandelt. Er ist als Gegenspieler Luthers zu sehen - begonnen hatte er jedoch als dessen Anhänger. Über Müntzers frühes Leben ist wenig bekannt, er stammte vermutlich aus einer stadtbürgerlichen Familie. Erst mit seiner Immatrikulation 1506 in Leipzig bzw. 1512 in Frankfurt gibt es einen gesicherten Anhaltspunkt. Das Studium brach er, vermutlich aus beruflichen Gründen, ab. Danach verdingte er sich als Lehrer, bis er um 1514 die Priesterweihe erhielt. Er nahm ein Altarpfrund ${ }^{35}$ in Braunschweig an und hatte nun intensiven Kontakt zu angesehenen Familien, die frühreformatorische Ansichten pflegten. Zwischen 1517 und 1519 sind Besuche in Wittenberg nachgewiesen. Schon in dieser Zeit formulierte er Kirchenkritik und befasste sich intensiv mit Humanismus und Mystik. 1521 erhielt er erstmals die Gelegenheit, vor einer großen Menge zu predigen. Seine Ansichten entfachten einen heftigen Streit mit den konservativen Kräften der Stadt, dieser mündete in seiner Entlassung. Von da an ging er zuerst nach Böhmen und dann auf Wanderschaft. Stationen waren wahrscheinlich Jena, Weimar und Erfurt. 1523 wurde Müntzer wieder sesshaft und betreute, diesmal in deutscher Sprache, die Allstädter Gemeinde der Johanneskirche als Prediger. In dieser Zeit wird erstmals deutlich, wie sich Müntzer von Luther theologisch abgrenzte: Er entwickelte eine zunehmend ablehnende Haltung gegenüber dem Adel und hielt immer politischere Reden. Dabei argumentierte er für den Einsatz von Gewalt, da gegenüber Gottlosen dieses Mittel erlaubt wäre. ${ }^{36}$

Nach der Zerstörung einer nahegelegenen Kapelle beschuldigten Müntzers Gegner ihn und seine Anhänger dieser Handlung, was zu seiner Entlassung führte. In Mühlhausen ist dann seit Mitte August 1524 seine Anwesenheit wieder nachweisbar. Er wurde freundlich aufgenommen und begann dort, den Gottesdienst zu reformieren und in deutscher Sprache zu halten. Auch hier ließ Streit nicht lange auf sich warten und in Folge von Unruhen wurden elf Artikel verbreitet. Diese dürften von Müntzer bearbeitet worden sein. Dies mündete sogar in der Gründung eines militärischen Bundes, der allerdings scheiterte. Anfang Oktober musste Müntzer mit anderen Beteiligten die Stadt verlassen. Zeitgleich begannen im Südwesten die ersten Unruhen der Bauernkriege. Seine Reaktionen darauf sind nicht bekannt, wohl aber, dass er sich jetzt immer mehr von Luthers Reformationsideen absetzte und diese zu kritisieren begann. In den Bäuerinnen und Bauern sah er die Gewalt Gottes, die sein Werk durchsetzen müssten, da

\footnotetext{
34 Scheidegger, Bauern \& Bauernkrieg.

35 Geistliches Amt mit den daraus resultierenden Einkünften: Jacob Grimm/Wilhelm Grimm, Deutsches Wörterbuch, Bd. 13, Leipzig 1889, Sp. 1799-1801.

36 Armin Gebhardt, Thomas Müntzer. Revolution statt Reformation. Eine Studie, Marburg 2004, S. 29.
} 
es die Adeligen nicht taten. Dies alles geschah unter dem Eindruck, sich bereits in der Endzeit zu befinden und die Welt auf die erneute Ankunft Christis vorzubereiten. ${ }^{37}$

Radikal war seine Vorstellung, die Dualität von weltlicher und geistiger Macht aufzulösen und so eine komplette Systemänderung zu erreichen. Diese Revolution sollte von Mühlhausen ausgehen. Müntzer überschätzte aber die militärischen Bedrohungen, die der Reichstadt von verschiedenen Seiten drohten. Nicht nur braute sich Unruhe durch das mögliche Eingreifen von ehemaligen Schutzmächten der Stadt zusammen, die der Reformation nicht positiv gegenüberstanden, sondern auch durch den theologischen Schlagabtausch von Müntzer und Luther. Noch war aber kein Angriff gestartet worden. Bis jetzt ließen sich die gewalttätigen Ereignisse als Notwehr zusammenfassen. ${ }^{38}$ Während sich rund um das Gebiet langsam die Aufstände ausbreiteten, wuchs die Kampfesstimmung, aber erst gegen Ostern 1525 eskalierte sie. Rasant wurde die Ordnung der Gesellschaft gestört. Während in anderen Gebieten darauf mit Furcht reagiert wurde, freute sich Müntzer über die Ereignisse. Schon früher hatte er, im Gegensatz zu Luther, eine theologische Erlaubnis zum Aufruhr formuliert. So wurde Mühlhausen schnell zu einem Zentrum für Aufständische. ${ }^{39}$

Während immer mehr Sympathisant*innen eintrafen, änderte Müntzer seine theologische Argumentation ab. Zuvor war es um eine innere Vorbereitungszeit für die Veränderung der Welt gegangen. Jetzt kehrte sich diese nach außen. Auch die reale Welt müsse umgestaltet werden, damit der Glaube ans Ziel gelangen könne. Solange der Mensch und seine Existenz bedroht wären, könne der wahre Glaube nicht entstehen. ${ }^{40}$ So erhielt der Konflikt in Mühlhausen eine ganz andere theologische Dimension als in anderen Orten. Mit April wurde die Situation dann gewalttätig: Während zuvor die Musterung und Ansammlung von Truppen im Vordergrund gestanden hatten, wurden nun das Rathaus belagert und die Stadttore besetzt. Anschließend stürmten die Truppen das nahegelegene Kloster und vertrieben die Geistlichen. Der Mob wurde danach noch größer und plünderte das Gebiet weiter. Die Verhandlungsbasis war durch diese Ausbrüche der Gewalt geschwächt. Weitere Exzesse der Brutalität, Brandstiftung und Zerstörung folgten. Gerade in dieser Situation wurde der Bewegung die theologische Legitimation, nicht nur von wissenschaftlicher Seite, abgesprochen. Viele Historiker*innen deuten die Situation in der Literatur als Klassenkampf. ${ }^{41}$ Tatsächlich scheint es viele Indizien zu geben, die für diese These sprechen, wie etwa die Änderung jener theologischen Begründung, die Gewalt leichter zulässt. Während es in anderen Gebieten darum ging, die Lage von bestimmten Schichten zu verbessern, aber das System nicht komplett zu verändern, ging es in Mühlhausen um eine radikale Kehrtwendung.

37 Günter Vogler, Müntzer, Thomas, in: Deutsche Biographie, O. D., [https://www.deutsche-biographie.de/sfz67128. html], eingesehen 18.6.2019.

38 Hans-Jürgen Goertz, Thomas Müntzer. Revolutionär am Ende der Zeiten. Eine Biographie, München 2015, S. 197199.

39 Ebd., S. 199-202.

40 Ebd., S. 203.

41 Ebd., S. 203-206. 


\section{Raum, Reformation und Bauernkrieg}

So wie es verschiedene individuelle Meinungen im Lager der Reformation gab, so muss immer die räumliche Dimension mitgedacht werden, denn die unterschiedlichen Standpunkte waren dadurch beeinflusst. Der Verlauf der Bauernbewegungen ging 1525 über Schwaben hinaus, von Franken bis nach Thüringen. In Oberschwaben konnte schon im April ein Vertrag zwischen Obrigkeit und Aufständischen geschlossen werden. Weniger rechtlich abgesichert ging es in Thüringen zu, wo Thomas Müntzer schließlich starb. Auch im heutigen Salzburger Raum, im Elsass, Franken, Tirol, Sachsen und Kärnten kam es zu Aufständen. ${ }^{42}$ Bayern, der Norden und der Nordosten des Reiches blieben hingegen unberührt. ${ }^{43}$

Natürlich gab es zwischen den verschiedenen Territorien Überlappungen. Es lassen sich nicht nur unterschiedliche Beweggründe für Gebiete ausmachen, sondern ebenso voneinander abweichende Intensitäten der Gewalt bzw. der Verhandlungen. Der Bauernkrieg 1525 verlief in Tirol anders als in Thüringen, Sachsen oder in Gebieten der Eidgenossenschaft. Auch die Agrarstruktur war mannigfaltig und nicht kohärent. Ebenso muss festgehalten werden, dass es etwa im Thüringer Raum sogar Schutz vor Willkür bei den Abgabenregelungen für Bäuerinnen und Bauern gab. Es waren also nicht alle der Macht der Obrigkeit schutzlos ausgeliefert. Dennoch gibt es gemeinsame Nenner, die die Bewegungen zusammenhielten. Neue Belastungen, Steuern und Verbote, das Wort Gottes nach dem Vorbild Luthers zu predigen, machten „dem gemeinen Mann" das Leben schwer. ${ }^{44}$ Doch auch oder vielmehr vor allem in Gebieten, in denen argumentiert werden könnte, die wirtschaftliche Situation der bäuerlichen Bevölkerung wäre gar nicht so schlecht gewesen, waren die Reformation und deren Lehren ein wichtiges Triebmittel. Gerade die endzeitliche Stimmung, die heraufbeschworen wurde, ließ die Situation eskalieren. ${ }^{45}$ Luther wollte die Aufstände zwar nicht, war aber mit seiner Lehre Schlüsselfigur und Motivator. Nicht nur Lehre und Wohlstand müssen als Faktoren gesehen werden, sondern auch die Strukturen der Gemeinden selbst. Diese waren äußerst vielfältig hinsichtlich Organisation und Partizipation. ${ }^{46}$ In einigen Gemeinden gab es schlicht keinen Bedarf, sich gegen die Obrigkeit aufzulehnen.

Wieder anders stellte sich die Situation in Tirol dar. Hier wurden die Zwölf Artikel vermutlich sogar noch rascher verbreitet als in den anderen Erblanden der Habsburger. Schon im Zeitraum zwischen Januar und Februar 1525 kam es zu Unruhen der Bergknappen gegen ihre Verwaltung. Doch erst die versuchte Hinrichtung eines Bauern führte zu Ausschreitungen. Ihnen schlossen sich Handwerker*innen und ein Teil des

42 Zur Mühlen, Reformation und Gegenreformation, S. 117-120.

43 Uwe Schirmer, Die Ursachen des Bauernkrieges in Thüringen. Eine sozial-, verfassungs- und reformationsgeschichtliche Spurensuche, in: Werner Greiling/Thomas Müller/Uwe Schirmer (Hrsg.), Reformation und Bauernkrieg (Quellen und Forschungen zu Thüringen im Zeitalter der Reformation 12), Köln 2019, S. 21-70, hier S. 39.

44 Schirmer, Ursachen des Bauernkrieges, S. 21-23.

45 Ebd., S. 26-36.

46 Peter Bierbrauer, Die ländliche Gemeinde im oberdeutsch-schweizerischen Raum, in: Peter Blickle (Hrsg.), Landgemeinde und Stadtgemeinde in Mitteleuropa. Ein struktureller Vergleich, München 1991, S. 169-190. 
Bürgertums an. Sie wählten einen Anführer, anschließend wurde der Aufstand durch Boten rasch verbreitet. Der Anführer, Michael Gaismair, erweiterte das Programm der Zwölf Artikel durch seine Veröffentlichung der 62 Meraner Artikel. Die hier enthaltenen Forderungen waren weitaus radikaler und können nicht mehr als reformatorisches Programm gesehen werden. Nachdem Gaismair eine Niederlage in Tirol einstecken musste, floh er zuerst in die Schweiz, wurde aber 1526 wieder aktiv, diesmal im Erzstift Salzburg. Hier war die Zusammensetzung des Aufstandes anders: Bäuerinnen und Bauern spielten eine eher untergeordnete Rolle. Außerdem war der Landesfürst ein Geistlicher. Munition für diesen Aufstand lieferte der dortige Zentralismus. Auch hier war es die öffentliche Empörung über eine Hinrichtung, die das Fass zum Überlaufen brachte. Zunächst wurde die Stadt Salzburg eingenommen und eine „Landschaft" gegründet. Hierbei ist interessant, dass sich ihr ebenfalls Adelige anschlossen. Es wurden eigene Artikel verfasst, die sich aber im Gegensatz zu denen aus Meran auf das Evangelium beriefen. In diesen gab es auch weltliche Forderungen, doch die ersten sieben richteten sich maßgeblich gegen die Missstände in der Kirche. ${ }^{47}$ Durch diesen recht knappen Einblick lässt sich schon ein Gefühl dafür bekommen, wie unterschiedlich die Zusammensetzung der Aufstände war. Dadurch erklären sich die verschiedenen Schwerpunkte bei den Forderungen, die dennoch vom roten Faden des Reformationsgedankens durchzogen sind.

Die reformatorischen Gedanken, die in den Bauernbewegungen aufgekommen waren, gingen aber nicht mit diesen unter. Sie lebten weiter und bildeten die Grundlage für spätere Aufstände: In Salzburg führte die Niederlage der Bäuerinnen und Bauern zu einer Vertiefung des neuen Evangeliumsverständnisses und auch in Tirol bildeten sich interessante religiöse Bewegungen. ${ }^{48}$ So etablierten sich die Wiedertäufer unter Hans Hut oder Balthasar Hubmaier. Letzterer stand zunächst in enger Verbindung mit Zwingli, bis ihre theologischen Differenzen zu groß wurden. ${ }^{49}$ Dieses Auseinanderdriften der reformatorischen Teilgruppen setzte sich jahrzehntelang fort und die Ergebnisse dieser Zersplitterung lassen sich noch heute konstatieren, etwa in den Täufergruppen, die im amerikanischen Raum die Zeit überdauerten. Die Konflikte lebten bis ins 17. Jahrhundert weiter und kamen 1618 mit dem Dreißigjährigen Krieg wieder an die Oberfläche, nämlich im oberösterreichischen Bauernkrieg von 1626. Ein Jahrhundert nach dem sogenannten deutschen Bauernkrieg widersetzte sich die Zivilbevölkerung der Einsetzung katholischer Pfarrer und den schweren Auflagen, die durch die Kriegssituation noch verschlimmert wurden.

47 Roland Schäffer, Der obersteirische Bauern- und Knappenaufstand und der Überfall auf Schladming 1525 (Militärische Schriftenreihe 62), Wien 1989, S. 9-11.

48 Peter Barton, Der vorweggenommene Bauernkrieg. Der Modellfall Innerösterreich, in: ders. (Hrsg.), Sozialrevolution und Reformation. Aufsätze zur Vorreformation, Reformation und zu den „Bauerkriegen“ in Südmitteleuropa, WienKöln-Graz 1975, S. 62-72, hier S. 64.

49 Bernd Moeller, Hubmaier, Balthasar in: Deutsche Biographie, o. D., [https://www.deutsche-biographie.de/ sfz34098.html], eingesehen 21.6.2019. 


\section{Fazit}

Zusammenfassend lässt sich feststellen, dass beide Entwicklungen - Bauernkrieg und Reformation - schon früh Verquickungen aufwiesen und ähnliche Ideen verbreiteten. Beide hatten ihre Ausgangspunkte im selben Jahrhundert und konnten durch dieselben Faktoren erstarken bzw. Durchsetzungskraft erhalten. Die eine Bewegung war jedoch zum Scheitern verurteilt, während die andere sich stabilisierte. ${ }^{50}$ Diese Arbeit machte im ersten Kapitel die parallele Entwicklung, welche die Reformation und die bäuerlichen Widerstände gemeinsam durchliefen, deutlich, ebenso die historiografischen Zuschreibungen und narrative Entwicklung, die das Thema erfuhr. Dies führte zu der nächsten Ebene, den persönlichen Verstrickungen und Meinungen der Zeitgenoss*innen zur Thematik. Das Herunterbrechen auf drei sehr verschiedene Persönlichkeiten erlaubte einen nuancierten Blick auf diese Verflechtungen und machte deutlich, welche unterschiedlichen und differenzierten Meinungen es gab. Danach folgte das Kapitel, welches sich mit der Frage der räumlichen Entwicklung beschäftigt. Dadurch wurde die Differenziertheit der gesellschaftlichen Ordnung, wirtschaftlichen Ausgangslagen und religiösen Grundhaltungen und Tendenzen verdeutlicht. Infolge dessen müssen die Fragen, die anfangs gestellt wurden, zur Überprüfung der formulierten Thesen noch einmal in Erinnerung gerufen werden.

(1) Inwieweit ist der Vorwurf zutreffend, die reformatorischen Ideen wären nur ein Vehikel gewesen, um wirtschaftliche Interessen der Bäuerinnen und Bauern durchzusetzen? (2) Welche Gemeinsamkeiten und Wechselwirkungen gab es zwischen Reformation und Bauernkrieg? So werden die Leser*innen bemerken, dass gerade die erste Frage und die zusammenhängende These bestätigt werden können. Zwar waren es auch ökonomische Faktoren, welche die bäuerliche Bevölkerung zum Aufstand brachten, doch muss die Rolle der Religion anders betrachtet werden als in der Gegenwart. Der christliche Glaube durchdrang alle Bevölkerungsschichten gleichermaßen auf eine intensive Art, die heute beinahe nicht mehr verstanden werden kann. Er spielte im Alltagsleben eine vollkommen andere Rolle als zum jetzigen Zeitpunkt. Deshalb wurde die nachfolgende These (1) weitestgehend bestätigt: Obwohl der Vorwurf, nur eine wirtschaftliche Strategie zu verfolgen, eine beliebte Argumentationslinie ist, kann zumeist die Wichtigkeit der reformatorischen Ideen nicht von der Hand gewiesen werden. Sie können nicht nur zweitrangig neben wirtschaftlichem Interesse stehen gelassen werden. Schwieriger wird es bei der zweiten These: Reformation und Bauernkrieg fungierten füreinander als Motor für Ideen und deren Umsetzung. Dafür ist das Kapitel, welches sich mit der personellen Ebene beschäftigt, ausschlaggebend. Prediger und Geistliche inspirierten mit ihren Aussagen, Auslegungen und Argumenten, waren Mediatoren im Konflikt zwischen Bevölkerungsgruppen, die sich diametral gegenüberstanden. Sie fungierten aber ebenso als Agitatoren und führten den Konflikt auf höhere Ebenen. Auch die Entwicklungen, die zu weiteren Spaltungen führten, sind dabei zu beachten. Hier kann die Argumentation sehr wohl zugelassen werden, dass die per-

50 Auch wenn zu betonen ist, dass es ein langwieriger Prozess war, bis tatsächlich eine Gleichstellung zwischen den Konfessionen erzielt war. 
sönliche Entwicklung der genannten Reformatoren während der Zeit des sogenannten deutschen Bauernkrieges eine Entwicklung war, welche die Reformation weiterführte.

Der Glaube an eine höhere Macht und die Aussicht auf ein besseres Leben lassen Menschen immer noch ins Extreme gehen. Es erscheint deshalb aus heutiger Sicht paradox, dass religiöse Beweggründe und wirtschaftliche Forderungen nicht unbedingt gleichrangig nebeneinanderstehen. Die Anordnung in den Zwölf Artikeln wurde von den Bäuerinnen und Bauern und deren Vertreter*innen bewusst gewählt und sollte ihren Standpunkt weiter verdeutlichen.

\section{Literatur und Quellen}

Althaus, Paul, Luthers Haltung im Bauernkrieg, Darmstadt 1971.

Amt der oberösterreichischen Landesregierung, Abteilung Kultur (Hrsg.), Der oberösterreichische Bauernkrieg 1626, Katalog zur Ausstellung im Linzer Schloss und im Schloss zu Scharnstein im Almtal von 14.05.1976-31.10.1976, Linz 1976.

Barton, Peter (Hrsg.), Sozialrevolution und Reformation. Aufsätze zur Vorreformation, Reformation und zu den Bauerkriegen in Südmitteleuropa, Wien-Köln-Graz 1975.

Ders., Der vorweggenommene Bauernkrieg. Der Modellfall Innerösterreich, in: ders. (Hrsg.), Sozialrevolution und Reformation. Aufsätze zur Vorreformation, Reformation und zu den „Bauernkriegen“ in Südmitteleuropa, Wien-Köln-Graz 1975, S. 62-72.

Beyer, Michael, Die drei Bauernkriegsschriften Martin Luthers von 1525, in: Werner Greiling/Thomas Müller/Uwe Schirmer (Hrsg.), Reformation und Bauernkrieg (Quellen und Forschungen zu Thüringen im Zeitalter der Reformation 12), Köln 2019, S. 241-258.

Bierbrauer, Peter, Die ländliche Gemeinde im oberdeutsch-schweizerischen Raum, in: Peter Blickle (Hrsg.), Landgemeinde und Stadtgemeinde in Mitteleuropa. Ein struktureller Vergleich, München 1991, S. 169-190.

Blickle, Peter, Der Bauernkrieg, München 20114.

Ders. (Hrsg.), Zwingli und Europa: Referate und Protokoll des internationalen Kongresses aus Anlass des 500. Geburtstages von Huldrych Zwingli vom 26.-30. März 1984, Göttingen 1985.

Ders., Die Reformation im Reich, Stuttgart $2000^{3}$.

Brendle, Franz, Das konfessionelle Zeitalter, Berlin 2010.

Flicker, Willi, Der oberösterreichische Bauernkrieg von 1626 in seinem politischen, konfessionellen, sozialen und kulturellen Umfeld, Dipl. Innsbruck 1991.

Furtwängler, Martin, Tagungsbericht. Bauernkrieg und Revolution. 200 Jahre Wilhelm Zimmermann - Ein Radikaler aus Stuttgart, 02.03.2007 Stuttgart, in: H-Soz-Kult, 11.04.2007, [www.hsozkult.de/conferencereport/id/tagungsberichte-1532], eingesehen 11.2.2020. 
Gebhardt, Armin, Thomas Müntzer. Revolution statt Reformation. Eine Studie, Marburg 2004.

Goertz, Hans-Jürgen, Thomas Müntzer. Revolutionär am Ende der Zeiten. Eine Biographie, München 2015.

Grimm, Jacob/Grimm, Wilhelm, Deutsches Wörterbuch, Bd. 13, Leipzig 1889.

Hamm, Berndt, Bürgertum und Glaube. Konturen der städtischen Reformation, Göttingen 1996.

Heiligsetzer, Georg, Der oberösterreichische Bauernkrieg 1626 (Militärhistorische Schriftenreihe Heft 32), Wien 1976.

Laube, Adolf/Seiffert, Hans Werner (Hrsg.), Flugschriften der Bauernkriegszeit, Wien 1978.

Luther, Martin, Eine schreckliche Geschichte und ein Gericht über Thomas Müntzer, in: Adolf Laube/Hans Werner Seiffert (Hrsg.), Flugschriften der Bauernkriegszeit, Wien 1978, S. 499-505.

Luther 2017, o. D., [https://www.luther2017.de/de/reformation/und-kultur/bild-bibel/ sola-scriptura/index.html], eingesehen 12.2.2020.

Lutz, Heinrich, Reformation und Gegenreformation (Oldenbourg Grundriss der Geschichte 10), München $1997^{4}$.

Lutz, Volker, Der Aufstand von 1596 und der Bauernkrieg von 1626 in und um Steyr (Veröffentlichung des Kulturamtes der Stadt Steyr Folge 33), Steyr 1976.

Mau, Wilhelm, Balthasar Hubmaier, Berlin-Leipzig 1912.

Maurer, Justus, Prediger im Bauernkrieg, Stuttgart 1979.

Moeller, Bernd, Hubmaier, Balthasar in: Deutsche Biographie, o. D., [https://www.deutsche-biographie.de/sfz34098.html], eingesehen 21.6.2019.

Mörke, Olaf, Die Reformation. Voraussetzungen und Durchsetzung (Enzyklopädie deutscher Geschichte 74), München 2005.

Müller, Thomas, Bauernkrieg in Thüringen. Eine kurze rezeptionsgeschichtliche Einführung, in: Werner Greiling/Thomas Müller/Uwe Schirmer (Hrsg.), Reformation und Bauernkrieg (Quellen und Forschungen zu Thüringen im Zeitalter der Reformation 12), Köln 2019, S. 9-20.

Oberman, Heiko, Die Reformation. Von Wittenberg nach Genf, Göttingen 1986.

Schäffer, Roland, Der obersteirische Bauern- und Knappenaufstand und der Überfall auf Schladming 1525 (Militärische Schriftenreihe 62), Wien 1989.

Scheidegger, Chr., Bauern \& Bauernkrieg, 2002, [https://www.zhref.ch/themen/reformationsjubilaeum/allgemeine-informationen/huldrych-zwingli/zwingli-lexikon-vona-bis-z-1/lexikon-b/bauern-bauernkrieg], eingesehen 17.6.2019. 
Schilling, Heinz, Die deutsche Gemeindereformation. Ein oberdeutsch-zwinglianisches Ereignis vor der "reformatorischen Wende" des Jahres 1525?, in: Zeitschrift für Historische Forschung 14 (1987), Heft 3, S. 325-332.

Schirmer, Uwe, Die Ursachen des Bauernkrieges in Thüringen. Eine sozial-, verfassungsund reformationsgeschichtliche Spurensuche, in: Werner Greiling/Thomas Müller/Uwe Schirmer (Hrsg.), Reformation und Bauernkrieg (Quellen und Forschungen zu Thüringen im Zeitalter der Reformation 12), Köln 2019, S. 21-70.

Schulze, Winfried, Deutsche Geschichte im 16. Jahrhundert. 1500-1618, Darmstadt 1997.

Sperling, Heinrich, Möglichkeiten zu sozialem und politischem Lernen in der deutschen Geschichte. Eine Untersuchung des Bauernkrieges, in: Reiner Wohlfeil (Hrsg,), Der Bauernkrieg 1524-26. Bauernkrieg und Reformation, München 1975, S. 246-279.

Vogler, Günter, Müntzer, Thomas, in: Deutsche Biographie, o. D., [https://www.deutsche-biographie.de/sfz67128.html], eingesehen 18.6.2019.

Wehler, Hans-Ulrich (Hrsg.), Der Deutsche Bauernkrieg 1524-1526 (Geschichte und Gesellschaft Sonderheft 1), Göttingen 1975.

Wohlfeil, Reiner (Hrsg.), Bauernkrieg 1524-25. Bauernkrieg und Reformation (Nymphenburger Texte zur Wissenschaft 21), München 1975.

zur Mühlen, Karl Heinz, Reformation und Gegenreformation, Göttingen 1999.

Zwingli, Ulrich, Von göttlicher und menschlicher Gerechtigkeit (30. Juli 1523), in: Emil Egli (Hrsg.), Huldreich Zwinglis sämtliche Werke, Bd, 2, Leipzig 1908, [http://www.irg. uzh.ch/static/zwingli-werke/index.php?n=Werk.21], eingesehen 17.6.2019.

Ders., Wer Ursache gebe zu Aufruhr usw. (7. Dezember resp. 28. Dezember 1524), in: Emil Egli (Hrsg.), Huldreich Zwinglis sämtliche Werke, Bd. 3, Leipzig 1914, [http://www. irg.uzh.ch/static/zwingli-werke/index.php?n=Werk.42], eingesehen 25.6.2019.

Sabrina Martina Pilsinger ist Studentin des Masterstudiengangs Geschichte und seit 2017 studentische Mitarbeiterin an der Universität Innsbruck.

sabrina.pilsinger@student.uibk.ac.at

\section{Zitation dieses Beitrages}

Sabrina Pilsinger, Reformation und Bauernkrieg, in: historia.scribere 12 (2020), S. $261-$ 276, [http://historia.scribere.at], eingesehen 15.6.2020 (=aktuelles Datum).

Creative Commons Licences 3.0 Österreich unter Wahrung der Urheberrechte der Autorlnnen. 